\title{
On-Surface Covalent Linking of Organic Building Blocks on a Bulk Insulator
}

\author{
Markus Kittelmann, ${ }^{\dagger}$ Philipp Rahe, ${ }^{\dagger}$ Markus Nimmrich, ${ }^{\dagger}$ Christopher M. Hauke, ${ }^{\dagger}$ André Gourdon, ${ }^{\ddagger}$ and \\ Angelika Kühnle ${ }^{\dagger, *}$ \\ ${ }^{\dagger}$ Institut für Physikalische Chemie, Johannes Gutenberg-Universität Mainz, Jakob-Welder-Weg 11, 55099 Mainz, Germany, and ${ }^{\ddagger}$ CNRS, CEMES, Nanoscience Group, \\ 29 Rue J. Marvig, 31055 Toulouse, France
}

M olecular self-assembly has been proven to constitute a powerful tool for creating tailor-made structures on surfaces. ${ }^{1}$ Upon tuning the subtle balance between intermolecular and molecule-surface interactions and by a rational design of the molecular building blocks, a multitude of structures have been constructed in clean and well-controlled ultrahigh-vacuum (UHV) conditions, ranging from perfect two-dimensional layers, unidirectional wire-like structures, ${ }^{2}$ and well-defined clusters ${ }^{3}$ to most complex architectures such as open networks ${ }^{4}$ and host-guest structures. ${ }^{5}$ Molecular self-assembly requires reversibility of the involved supramolecular interactions to reach the (local) thermodynamic minimum. ${ }^{6,7}$ This inherently required reversibility, however, constitutes a considerable drawback when aiming at chemically stable structures that bear the potential to be used ex situ in the harsh conditions of ambient environment. Moreover, when having molecular electronics applications in mind, the weak and reversible interactions pose a further challenge, as they hardly provide sufficient intermolecular electron transport capabilities. Only recently, the concept of on-surface synthesis $^{8-10}$ has been exploited as a promising strategy to overcome these limitations and obtain thermally and chemically stable structures by covalent bonding of suitable precursors directly on the substrate in UHV. ${ }^{11-18}$ This strategy presents several other important advantages such as the possibility to prepare large molecules impossible to synthesize in solution due to their low solubility or new reactions not observed in solution by 2D confinement of molecular precursors., ${ }^{9,18}$ So far, the few successful demonstrations of on-surface synthesis under UHV conditions have been limited to metallic substrates, ${ }^{11-17}$ and only in one instance has a monolayer

ABSTRACT On-surface synthesis in ultrahigh vacuum provides a promising strategy for creating thermally and chemically stable molecular structures at surfaces. The two-dimensional confinement of the educts, the possibility of working at higher (or lower) temperatures in the absence of solvent, and the templating effect of the surface bear the potential of preparing compounds that cannot be obtained in solution. Moreover, covalently linked conjugated molecules allow for efficient electron transport and are, thus, particularly interesting for future molecular electronics applications. When having these applications in mind, electrically insulating substrates are mandatory to provide sufficient decoupling of the molecular structure from the substrate surface. So far, however, onsurface synthesis has been achieved only on metallic substrates. Here we demonstrate the covalent linking of organic molecules on a bulk insulator, namely, calcite. We deliberately employ the strong electrostatic interaction between the carboxylate groups of halide-substituted benzoic acids and the surface calcium cations to prevent molecular desorption and to reach homolytic cleavage temperatures. This allows for the formation of aryl radicals and intermolecular coupling. By varying the number and position of the halide substitution, we rationally design the resulting structures, revealing straight lines, zigzag structures, and dimers, thus providing clear evidence for the covalent linking. Our results constitute an important step toward exploiting on-surface synthesis for molecular electronics and optics applications, which require electrically insulating rather than metallic supporting substrates.

KEYWORDS: on-surface synthesis · surface chemistry - covalent linking · bulk insulator $\cdot$ noncontact atomic force microscopy $\cdot$ molecular electronics

of $\mathrm{NaCl}(100)$ on $\mathrm{Ag}(100)$ been used. ${ }^{19}$ However, for many applications such as molecular electronics, phase-supported organic catalysis, or molecular optics, it would be exceedingly attractive to transfer this technique to bulk insulators to prevent electronic coupling and leakage or nonradiative quenching. Covalent linking of organic molecules on a bulk insulator surface, however, poses additional challenges: The preassembly of the molecular building blocks is frequently hampered by the comparatively weak moleculesurface interactions, leading to clustering at step edges and molecular bulk crystal formation. ${ }^{20}$ Moreover, thermal activation of a coupling reaction is not feasible on many insulating surface, such as alkali halides, as most organic molecules would desorb at *Address correspondence to
kuehnle@uni-mainz.de.

Received for review August 28, 2011 and accepted September 7, 2011.

Published online September 07, 2011 $10.1021 / \mathrm{nn} 2033192$

() 2011 American Chemical Society 
temperatures below the reaction temperature. Finally, in most cases the metallic substrate seems to have a catalytic role in the coupling reaction, ${ }^{21}$ raising the question as to whether similar reactions can be observed on an insulator. These are some of the reasons why on-surface synthesis has not been demonstrated on a bulk insulator so far.

Herein we present the covalent coupling of four different halide-substituted benzoic acids (for an overview see Figure 1), namely, 2,5-diiodobenzoic acid (DIBA), 2,5-dichlorobenzoic acid (DCBA), 3,5-diiodosalicylic acid (DISA), and 4-iodobenzoic acid (IBA) on a bulk insulator, namely, calcite. The carboxylic acid moiety is known to bind strongly toward calcite, ${ }^{22}$ thus, compared to other insulating surfaces, the carboxylic acid-calcite system has revealed very promising results in terms of substratetemplated molecular self-assembly. ${ }^{23}$ Here, the comparatively high molecule-substrate binding strength is employed to prevent clustering at step edges and to avoid desorption upon thermal activation of the coupling reaction. Our results indicate that the carboxylic acid group deprotonates before covalent linking. The presence of the carboxylate group, being a strong electron donor, is expected to weaken the phenyl-halide bond and, therefore, favor homolytic cleavage of this bond to give reactive phenyl radicals at moderate temperatures without the need of a coupling catalyst.

Upon deposition at room temperature (RT), DIBA and DCBA form extended and highly ordered monolayers, which undergo a clear transition into straight molecular wires upon thermal activation, as unraveled by noncontact atomic force microscopy (NC-AFM). The observed structures fit excellently in size with a model of a covalently bound, completely conjugated molecular row.

When changing the position of the halide substitution from 2,5 to 3,5 as in 3,5-diiodosalicylic acid, again a distinct structural transition can be observed upon thermal activation; however, in this case zigzag instead of straight structures are revealed. Finally, when having only one halide atom per molecule, dimers instead of extended rows are expected to form upon covalent linking. Indeed, thermal activation of 4-iodobenzoic acid results in the formation of dimer-like molecular structures that fit in size to the expected reaction product, 4,4-biphenyldicarboxylic acid (BPDCA).

Comparison with a benzoic acid of similar acidity but without the halide functionality (2,5-dihydroxybenzoic acid, DHBA) exhibits no intermolecular coupling and, therefore, provides further evidence for the specificity of the observed reactions.

\section{RESULTS AND DISCUSSION}

First, a submonolayer coverage of DIBA is deposited onto a freshly cleaved calcite (10-14) surface held at RT. After deposition, the resulting structure is observed by NC-AFM. An overview image is shown in Figure 2a,

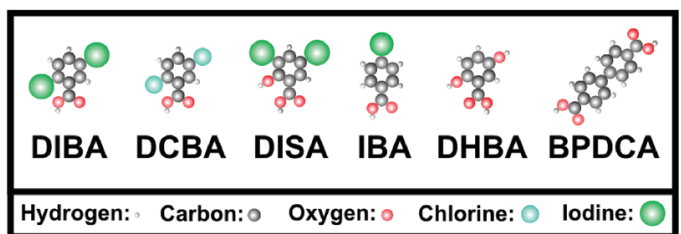

Figure 1. Models of the used organic molecules comprising 2,5-diiodobenzoic acid (DIBA), 2,5-dichlorobenzoic acid (DCBA), 3,5-diiodosalicylic acid (DISA), 4-iodobenzoic acid (IBA), 2,5-dihydroxybenzoic acid (DHBA), and 4,4-biphenyldicarboxylic acid (BPDCA).
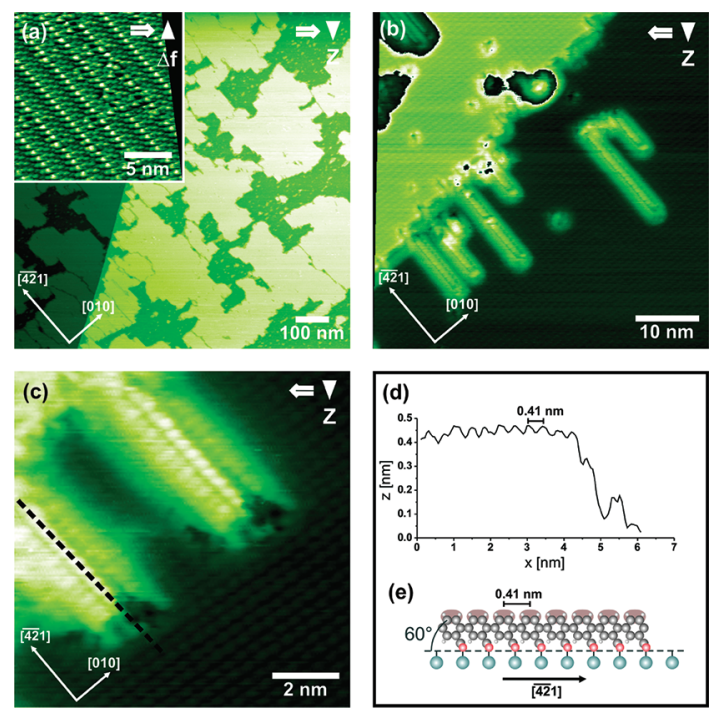

Figure 2. Covalent linking of DIBA. (a) Overview NC-AFM image of the as-deposited structure at RT, showing one monolayer high islands. Inset: Drift-corrected detailed NCAFM image, revealing a $(5 \times 1)$ unit cell. (b) NC-AFM image of DIBA after annealing above $530 \mathrm{~K}$ (here: $555 \mathrm{~K}$ ). The structures decorating step edges and rows are observed growing along the [ $\overline{4} \overline{2} 1]$ direction. (c) Detailed NC-AFM image, showing two molecular rows and the substrate with atomic resolution. (d) Height profile as indicated in Figure 1c, revealing a repeat distance of $0.41 \mathrm{~nm}$, in excellent agreement with a reacted molecular chain. (e) Model explaining the reacted structure.

revealing two calcite terraces that are covered by molecular islands with an apparent height of approximately $0.7 \mathrm{~nm}$. From this image it can be concluded that individual DIBA molecules are mobile and diffuse on the surface to form extended stable monolayers. A close-up of this structure exhibits a highly ordered structure as shown in the drift-corrected image ${ }^{24}$ presented in the inset in Figure 2a. A detailed analysis reveals a $(5 \times 1)$ superstructure exhibiting a moiré pattern (for details see Supplementary Figure S1). Molecular dynamics calculations ${ }^{22}$ suggest that molecules containing a carboxylic acid moiety anchor to the calcite surface with the carboxylic group binding to a surface oxygen and calcium atom in an upright fashion. However, the binding is of course greatly influenced by the protonation or deprotonation state of the acid. For DIBA, having a calculated $\mathrm{p} K_{\mathrm{a}}$ of 2.51 
(i.e., in vacuo), our results comparing benzoic acids with different acidity indicate that DIBA molecules are already deprotonated at RT. We note that the listed $\mathrm{p} K_{\mathrm{a}}$ values must not be taken too literally, as adsorption onto the surface might have a significant influence. However, the general trend in acidity should be reflected correctly by considering the calculated (in vacuo) values. Thus, in the case of DIBA we expect the negatively charged carboxylate group to anchor to the calcium ions. This is in agreement with the abovedrawn conclusion of upright-standing molecules. As for the deprotonation of other carboxylic acids on surfaces, we cannot clarify the question as to whether the dissociated hydrogen atoms remain on the surface or not. However, it appears likely that the protons bind to the oxygen atoms of the carbonate group.

When annealing the DIBA sample to a temperature above $530 \mathrm{~K}$, a completely different situation is present, as shown in Figure 2b. Now, in contrast to what was observed before at RT, no extended islands are formed, but rows running along the $[\overline{4} \overline{2} 1]$ direction. The rows shown in Figure $2 \mathrm{~b}$ decorate the step edges (one step edge is running from the lower left to the upper right part of the image). This drastic change in molecular structure is explained by thermolytic dissociation of the two iodine atoms from the molecule and subsequent covalent linking of the radical molecules. As the iodine atoms are substituted at the 2 and 5 positions, i.e., at opposite positions, a straight row is expected upon covalent linking. A detailed view given in the drift-corrected image in Figure $2 c$ reveals a highly ordered structure that fits in size with molecules forming a row after iodine dissociation and reaction of radicals. From a height profile taken at a line indicated in Figure $1 \mathrm{c}$, a repeat distance of $0.41 \mathrm{~nm}$ is revealed (Figure $2 \mathrm{~d}$ ). This distance is in excellent agreement with a model of a reacted, completely conjugated chain as shown in Figure 2e. In this model, the molecule is again anchored toward the surface with the carboxylate groups as discussed before. In order to allow for covalent bond formation at the previous iodine positions, the molecules have to tilt at an angle of approximately $60^{\circ}$ between the main molecular axis and the surface. This tilt results in a calculated reduction of the apparent height from nominally $0.75 \mathrm{~nm}$ for the iodine-free molecules to $0.65 \mathrm{~nm}$. Experimentally, we reveal an apparent height of $0.45 \mathrm{~nm}$, which is somewhat smaller than the expected value. However, we need to stress that different interaction forces greatly influence height measurements in NC-AFM, readily explaining this height difference.

The same reaction pathway is expected for DCBA, where the iodine atoms are replaced by chlorine atoms. DCBA has basically the same $\mathrm{p} K_{\mathrm{a}}$ value of 2.50 , but a higher carbon-halide bond strength ( $\mathrm{C}-\mathrm{Cl}: 397$ $\mathrm{kJ} / \mathrm{mol}$ versus $\mathrm{C}-\mathrm{I}: 268 \mathrm{~kJ} / \mathrm{mol}$ ). On the basis of the binding energies alone, very high thermolytic cleavage
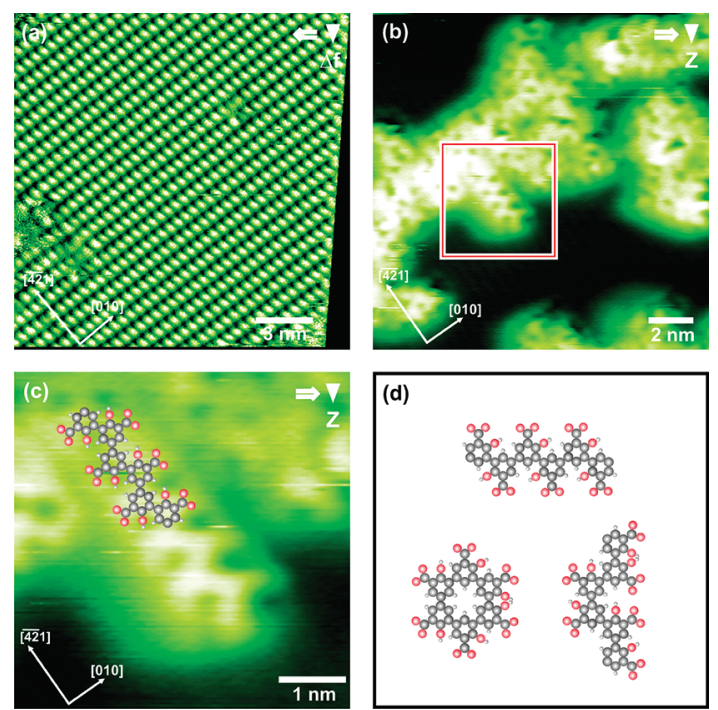

Figure 3. Covalent linking of DISA. (a) NC-AFM image of DISA deposited onto the calcite surface held at RT, revealing a commensurate $(1 \times 1)$ structure. (b) Detailed NC-AFM image of the sample after annealing to $580 \mathrm{~K}$, revealing a distinctly different molecular structure. A zigzag pattern is obtained as marked by a rectangle. (c) Close-up of the marked area in (b) with a superimposed model of the covalently linked molecules. (d) Model of the different structures possible upon covalent linking. Besides a zigzag row, also other, kinked and circular patterns are possible.

temperatures would be expected. Interestingly, in a recent study, thermolytic cleavage of bromine has been achieved at $590 \mathrm{~K}$ within a molecule sublimator (i.e., in the absence of a metallic surface) ${ }^{17}$ In our case, as mentioned above, the carboxylate formation and the interaction with the surface are expected to facilitate the homolytic cleavage. Thus, exclusively considering the bond strengths certainly oversimplifies the situation. We do, however, expect that somewhat higher temperatures are required when repeating the same experiments with DCBA instead of DIBA. We have done this experiment (not shown) and, indeed, find the same structures, but at an increased transition temperature, which has to be raised above $565 \mathrm{~K}$ for inducing covalent linking, supporting the conclusion above.

After annealing, we observe an increased defect density in the case of both DIBA and DCBA. These defects might arise from the dissociated iodine and chlorine atoms, respectively. However, we speculate that most of the dissociated iodine and chlorine atoms desorb upon annealing.

One may speculate that the structural changes observed are due only to a deprotonation of the carboxylic acid, which might be accompanied with a structural change. In order to exclude this possibility, we have checked the structures formed by DHBA, where the halide atoms are replaced by hydroxyl groups. DHBA has a $\mathrm{p} K_{\mathrm{a}}$ of 2.97 , which is somewhat higher than the $\mathrm{p} K_{\mathrm{a}}$ of the two acids tested above. Right after deposition, we observe a coexistence of two 
different structures, one of which is a transient structure that transform with time (and after annealing to $440 \mathrm{~K})$ into the more stable structure, exhibiting a $(1 \times 5)$ unit cell. We tentatively ascribe this transition to the deprotonation step (see Supplementary Figure S2 and Table S1). Upon annealing this structure to $520 \mathrm{~K}$, the ordered structure vanishes, leaving behind an unordered structure of probably fragmented molecules. This structure remains on the surface for all temperatures accessible. Most importantly, no structure is found that resembles the molecular row structure as observed before in the cases of DIBA and DCBA. This is a further confirmation for the specifity of the above shown covalent linking.

Next, we have changed the substitution position from 2,5 to 3,5 with the objective to create a zigzag structure after covalent linking. As 3,5-diiodobenzoic acid was not available for this study, 3,5-diiodosalicylic acid is used instead, having a $\mathrm{p} K_{\mathrm{a}}$ of 2.07 . This low $\mathrm{p} K_{\mathrm{a}}$ value suggests the molecules to be already deprotonated at RT. The as-deposited structure is shown in Figure $3 a$, revealing a commensurate $(1 \times 1)$ superstructure with an apparent height of $0.6 \mathrm{~nm}$, which agrees with upright-standing molecules. Annealing this sample to $580 \mathrm{~K}$ again results in a distinctly different molecular pattern, as shown in Figure $3 \mathrm{~b}$. In this image, molecular features of differing shape are observed. The apparent height of these structures is on the order of $0.35 \mathrm{~nm}$, suggesting flat-lying molecules in this case. Among them, zigzag structures are, indeed, obtained, as marked by a rectangle in the center of the image. An enlarged view is given in Figure $3 c$ along with a model of covalently linked poly(metaphenyl) molecules forming a zigzag row. The observed transition from standing molecules at RT to lying molecular structures upon annealing can be readily understood by considering the position of the carboxylate groups. In the case of individual DISA molecules, all molecules can arrange with the carboxylate groups pointing toward the calcite surface, resulting in a layer of upright-standing molecules. After reaction, however, the carboxylate groups point in opposite directions, which prevents concerted binding of these groups to the surface, explaining the transition from standing to flatlying molecules. Besides zigzag structures, DISA can form other patterns, which occur when the molecules link with a different angle, as illustrated in Figure 3d. This fact readily explains the less ordered appearance of DISA upon annealing as compared to the structures observed after annealing of DIBA, as only straight lines are possible in the latter case. The structural change from straight lines as observed for 2,5-substituted DIBA and DCBA to aggregates with a kinked linking in the case of 3,5-substituted DISA constitutes a further strong confirmation of the above-drawn conclusion of covalent linking.
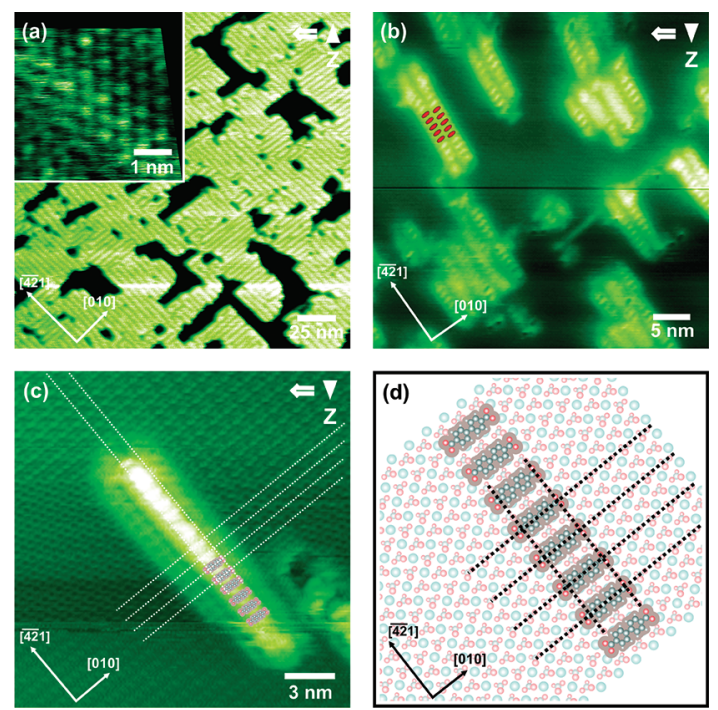

Figure 4. Covalent linking of IBA. (a) Upon moderate annealing of the IBA-covered sample to a temperature of $520 \mathrm{~K}$, two domains are revealed, exhibiting a large-scale moiré modulation. Inset: Close-up of one of the domains, exhibiting a $(8 \times 1)$ unit cell. (b) Molecular structure of the IBA-covered substrate after annealing to $580 \mathrm{~K}$ showing molecular rows running along the [ $\overline{4} \overline{2} 1]$ direction. Two or more rows align, resulting in double rows and larger arrangements. The rows are composed of individually resolved features that are aligned side-by-side. Ellipses having the size of the expected reaction product, BPDCA, are superimposed onto the row. (c) Close-up of a single molecular row with superimposed BPCDA model molecules. Due to a tip-convolution effect, the row is imaged with a "halo", which is a known artifact in AFM imaging. Along the [010] direction, lines are drawn that superimpose with the bright calcite features (protruding oxygen atoms of one type of carbonate group). Along the [ $\overline{4} \overline{2} 1]$ direction, the lines are superimposed onto the less bright features (protruding oxygen atoms of the opposite type of carbonate group). (d) Structural model showing the flat-lying molecules with the carboxylate groups adsorbed on top of two calcium ions of the substrate.

As a further evidence for the coupling reaction, we next discuss reducing the number of halide atoms at the phenyl ring from two to one. Molecules with only one halide atom are expected to form dimers instead of extended wires upon covalent linking. To follow this approach, 4-iodobenzoic acid with a $\mathrm{pK}_{\mathrm{a}}$ of 4.02 is deposited onto freshly cleaved calcite. Due to the comparatively high $\mathrm{p} K_{\mathrm{a}}$ value, the molecules remain protonated at RT, as is evident from a further structural transition observed upon moderate annealing to $520 \mathrm{~K}$ before inducing covalent coupling at higher temperatures as explained in the following.

At RT, extended two-dimensional islands with an apparent height of $0.5 \mathrm{~nm}$ and an internal hexagonal ordering are revealed (see Supplementary Figure S3). Upon moderate annealing at $520 \mathrm{~K}$, a first structural change is observed. After this annealing step, two domains of extended islands are observed; however, the apparent height increases to $0.8 \mathrm{~nm}$ (see Figure 4a). A highly ordered structure can be resolved within the domains (see inset in Figure $4 a)$, revealing a $(8 \times 1)$ unit 
cell. The change in apparent height suggests a transition from lying to upright-standing molecules upon moderate annealing. This structural change is ascribed to the deprotonation of the molecules, taking place at higher temperatures as compared to the three other benzoic acids discussed above. This can be understood by comparing the $\mathrm{p} K_{\mathrm{a}}$ values (4.02 versus 2.51 (DIBA), 2.50 (DCBA), and 2.07 (DISA)). Thus, the molecules are assumed to bind as negatively charged carboxylates (for details, see Supplementary Table S1). In an attempt to deiodize the IBA molecules and to induce a covalent linking of the remaining radical molecules to form biphenyldicarboxylic acid molecules, the molecules are further annealed to thermally activate the deiodization and subsequent linking. Annealing an IBA-covered calcite surface to $580 \mathrm{~K}$ again results in a significant structural change. As shown in Figure $4 b$, now rows are obtained that are oriented along the $[\overline{4} \overline{2} 1]$ calcite direction. The apparent height of the rows is $0.4 \mathrm{~nm}$, suggesting flat-lying molecules. The rows are composed of features that fit excellently in size with BPDCA molecules aligned side-by-side, as illustrated by the superimposed ellipses with the size of BPDCA molecules (Figure 4b). Often, two or more rows are observed to align, resulting in double rows and larger arrangements. However, single rows exist as well, as shown in Figure 4c. On the basis of these data, which reveal both the molecular rows and the calcite lattice simultaneously, the molecule adsorption position can be determined unambiguously. The calcite lattice reveals pronounced reconstructions, a $(2 \times 1)$ reconstruction and the so-called row pairing, allowing for an identification of the lattice sites. ${ }^{25}$ The lines drawn along the [010] direction in Figure $4 \mathrm{c}$ are superimposed onto the bright features of the calcite surface, which are ascribed to the protruding oxygen atoms of carbonate groups having the same orientation. The lines in the $[\overline{4} \overline{2} 1]$ direction are placed in a way that they superimpose the less bright features, which are known to be the oxygen atoms of the carbonate groups having the opposite orientation within the unit cell. As can be seen, the molecular row is centered in between the lines drawn in the [ $\overline{4} \overline{2} 1]$ direction. The molecules within the row are centered on the lines superimposed along the [010] direction. With this information, a precise structural model can be constructed, as given in Figure $4 \mathrm{~d}$. In this model, we assume the reacted IBA molecules to be deprotonated, as suggested by the structural change observed upon moderate annealing. The size of a deprotonated BPDCA molecule allows for an excellent alignment of the negatively charged carboxylate groups on top of two calcium ions of the substrate, which is exactly what we obtain from the model. The measured molecular spacing along the $[\overline{4} \overline{2} 1]$ direction of $0.81 \mathrm{~nm}$ reflects the spacing of every two calcium ions along this direction. Along the [010] direction, the measured spacing of $2.07 \mathrm{~nm}$ between adjacent rows again reflects the calcite lattice dimensions (four times $0.51 \mathrm{~nm}$ ) and, thus, further supports the assumption of a welldefined adsorption position in the case of covalently linked IBA molecules. We take the excellent agreement in size and the very reasonable spacing with respect to the calcite substrate as strong indications for the reaction of IBA to deprotonated BPDCA. Moreover, the transition in the molecular orientation from standing upright to lying down provides further evidence for the covalent linking. After the reaction, the carboxylic groups point in opposite directions, making concerted binding of the carboxylate groups to the calcite impossible. As a consequence, the dimers are expected to lie flat on the surface, which is, indeed, observed. Finally, a direct comparison with BPDCA molecules further reveals the structural similarity of the reacted IBA and as-deposited BPDCA molecules (see Supplementary Figure S4), serving as a further confirmation of the above-drawn conclusion.

\section{CONCLUSION}

To conclude, we have demonstrated the covalent linking of halide-substituted benzoic acid molecules by thermal activation on a bulk insulator, namely, the (104) cleavage plane of calcite. The resulting products constitute conjugated molecular structures on a truly insulating support, providing electronic decoupling from the substrate, which is mandatory for future applications. By comparison of different substitution positions as well as changing the number of halidesubstituted atoms, we demonstrate the specificity of this approach. Our results pave the way for application of on-surface synthesis on bulk insulators.

\section{METHODS}

Calcite Preparation. Optical quality calcite samples from Korth Kristalle $\mathrm{GmbH}$ (Kiel, Germany) are cleaved in situ, resulting in flat (104) cleavage planes. ${ }^{26}$ Right after cleavage, the crystals are heated to $480 \mathrm{~K}$ for about one hour to remove surface charges.

Molecule Deposition. The molecules were purchased from Aldrich (Munich, Germany) and thoroughly outgassed at RT for $60 \mathrm{~h}$ prior to use. All molecules are sublimated in situ onto the freshly prepared calcite surface using home-built Knudsen cells.

NC-AFM Imaging. All experiments are carried out under UHV conditions (base pressure $\leq 1 \times 10^{-10}$ mbar) using a VT AFM 25 atomic force microscope (Omicron, Taunusstein, Germany) operated in the frequency modulation noncontact mode (NCAFM). The system is equipped with an easyPLL Plus controller and phase-locked loop detector (Nanosurf, Liestal, Switzerland) 
for oscillation excitation and signal demodulation. We use n-doped silicon cantilevers (NanoWorld, Neuchâtel, Switzerland) with resonance frequencies of around $300 \mathrm{kHz}$ (type PPP-NCH) excited to oscillation amplitudes of about $10 \mathrm{~nm}$. Prior to their use, the cantilevers were $\mathrm{Ar}^{+}$sputtered at $2 \mathrm{keV}$ for $5 \mathrm{~min}$ to remove contaminants. Depending on the feedback loop settings, either frequency shift $(\Delta f)$ or topography $(z)$ images are presented here. For the $\Delta f$ images, the distance feedback loop was set very slow in order to provide quasi constant-height images while still following the overall tilt of the sample surface. Image type as well as fast and slow scan directions are given in the upper right corner in each image. The images are displayed such that bright corresponds to high attractive interactions while dark corresponds to less attractive or even repulsive interactions. ${ }^{27}$

Thermal Activation Step. The molecules are thermally activated by annealing the calcite substrate with a pyrolytic boron nitride heater positioned underneath the sample. The temperature is controlled by a thermocouple mounted at the sample stage about $2.5 \mathrm{~cm}$ apart from the sample. The temperature specifications given here are the corresponding temperatures expected at the calcite sample based on an individual calibration curve supplied by the manufacturer (Omicron, Taunusstein, Germany).

Acknowledgment. A.K. acknowledges support by the German Research Foundation (DFG) through the SFB 625 grant TP B17.

Supporting Information Available: Further NC-AFM images as mentioned throughout the text. This material is available free of charge via the Internet at http://pubs.acs.org.

\section{REFERENCES AND NOTES}

1. Barth, J. V. Molecular Architectonic on Metal Surfaces. Annu. Rev. Phys. Chem. 2007, 58, 375-407.

2. Barth, J. V.; Weckesser, J.; Cai, C.; Günter, P.; Bürgi, L.; Jeandupeux, O.; Kern, K. Building Supramolecular Nanostructures at Surfaces by Hydrogen Bonding. Angew. Chem., Int. Ed. 2000, 39, 1230-1234.

3. Böhringer, M.; Morgenstern, K.; Schneider, W.-D.; Berndt, R.; Mauri, F.; Vita, A. D.; Car, R. Two-Dimensional SelfAssembly of Supramolecular Clusters and Chains. Phys. Rev. Lett. 1999, 83, 324-327.

4. Pawin, G.; Wong, K. L.; Kwon, K. Y.; Bartels, L. A Homomolecular Porous Network at a Cu(111). Surf. Sci. 2006, 313, 961-962.

5. Theobald, J. A.; Oxtoby, N. S.; Phillips, M. A.; Champness, N. R.; Beton, P. H. Controlling Molecular Deposition and Layer Structure with Supramolecular Surface Assemblies. Nature 2003, 424, 1029-1031.

6. Kühnle, A. Self-Assembly of Organic Molecules at Metal Surfaces. Curr. Opin. Colloid Interface Sci. 2009, 14, 157168

7. Whitesides, G. M.; Mathias, J. P.; Seto, C. T. Molecular SelfAssembly and Nanochemistry: A Chemical Strategy for the Synthesis of Nanostructures. Science 1991, 254, 1312-19.

8. Gourdon, A. On-Surface Covalent Coupling in Ultrahigh Vacuum. Angew. Chem., Int. Ed. 2008, 47, 6950-6953.

9. Franc, G.; Gourdon, A. Covalent Networks through onSurface Chemistry in Ultra-High Vacuum: State-of-the-Art and Recent Developments. Phys. Chem. Chem. Phys. 2011, 13, 14283-14292.

10. Perepichka, D. F.; Rosei, F. Extending Polymer Conjugation into the Second Dimension. Science 2009, 323, 216-217.

11. Cai, J. M.; Ruffieux, P.; Jaafar, R.; Bieri, M.; Braun, T.; Blankenburg, S.; Muoth, M.; Seitsonen, A. P.; Saleh, M.; Feng, X. L.; et al. Atomically Precise Bottom-up Fabrication of Graphene Nanoribbons. Nature 2010, 466, 470-473.

12. Schmitz, C. H.; Ikonomov, J.; Sokolowski, M. Two-Dimensional Ordering of Poly(p-Phenylene-Terephthalamide) on the $A g(111)$ Surface Investigated by Scanning Tunneling Microscopy. J. Phys. Chem. C 2009, 113, 11984-11987.

13. Lipton-Duffin, J. A.; Ivasenko, O.; Perepichka, D. F.; Rosei, F. Synthesis of Polyphenylene Molecular Wires by SurfaceConfined Polymerization. Small 2009, 5, 592-597.
14. Weigelt, S.; Busse, C.; Bombis, C.; Knudsen, M. M.; Gothelf, K. V.; Laegsgaard, E.; Besenbacher, F.; Linderoth, T. R. Surface Synthesis of 2D Branched Polymer Nanostructures. Angew. Chem., Int. Ed. 2008, 47, 4406-4410.

15. Zwaneveld, N. A. A.; Pawlak, R.; Abel, M.; Catalin, D.; Gigmes, D.; Bertin, D.; Porte, L. Organized Formation of 2D Extended Covalent Organic Frameworks at Surfaces. J. Am. Chem. Soc. 2008, 130, 6678-6679.

16. Matena, M.; Riehm, T.; Stöhr, M.; Jung, T. A.; Gade, L. H. Transforming Surface Coordination Polymers into Covalent Surface Polymers: Linked Polycondensed Aromatics through Oligomerization of N-Heterocyclic Carbene Intermediates. Angew. Chem., Int. Ed. 2008, 47, 2414-2417.

17. Grill, L.; Dyer, M.; Lafferentz, L.; Persson, M.; Peters, M. V.; Hecht, S. Nano-Architectures by Covalent Assembly of Molecular Building Blocks. Nat. Nanotechnol. 2007, 2, 687-691.

18. Mendez, J.; Lopez, M. F.; Martin-Gago, J. A. On-Surface Synthesis of Cyclic Organic Molecules. Chem. Soc. Rev. 2011, 40, 4578-4590.

19. Abel, M.; Clair, S.; Ourdjini, O.; Mossoyan, M.; Porte, L. Single Layer of Polymeric Fe-Phthalocyanine: An Organometallic Sheet on Metal and Thin Insulating Film. J. Am. Chem. Soc. 2011, 133, 1203-1205.

20. Kunstmann, T.; Schlarb, A.; Fendrich, M.; Wagner, T.; Möller, R.; Hoffmann, R. Dynamic Force Microscopy Study of 3,4,9,10-Perylenetetracarboxylic Dianhydride on Kbr(001). Phys. Rev. B 2005, 71, 121403.

21. Gutzler, R.; Walch, H.; Eder, G.; Kloft, S.; Heckl, W. M.; Lackinger, M. Surface Mediated Synthesis of 2D Covalent Organic Frameworks: 1,3,5-Tris(4-Bromophenyl)Benzene on Graphite(001), Cu(111), and Ag(110). Chem. Commun. 2009, 4456-4458.

22. Duffy, D. M.; Harding, J. H. Modelling the Interfaces between Calcite Crystals and Langmuir Monolayers. J. Mater. Chem. 2002, 12, 3419-3425.

23. Rahe, P.; Nimmrich, M.; Greuling, A.; Schütte, J.; Stará, I. G.; Rybáček, J.; Huerta-Angeles, G.; Starý, I.; Rohlfing, M.; Kühnle, A. Toward Molecular Nanowires Self-Assembled on an Insulating Substrate: Heptahelicene-2-Carboxylic Acid on Calcite (10-14). J. Phys. Chem. C 2010, 114, 1547-1552.

24. Rahe, P.; Bechstein, R.; Kühnle, A. Vertical and Lateral Drift Corrections of Scanning Probe Microscopy Images. J. Vac. Sci. Technol. B 2010, 28, C4E31-C4E38.

25. Schütte, J.; Rahe, P.; Tröger, L.; Rode, S.; Bechstein, R.; Reichling, M.; Kühnle, A. Clear Signature of the $(2 \times 1)$ Reconstruction of Calcite (10-14). Langmuir 2010, 26, 8295-8300.

26. Tröger, L.; Schütte, J.; Ostendorf, F.; Kühnle, A.; Reichling, M. Concept for Support and Cleavage of Brittle Crystals. Rev. Sci. Instrum. 2009, 80, 063703.

27. Rahe, P.; Bechstein, R.; Schütte, J.; Ostendorf, F.; Kühnle, A. Repulsive Interaction and Contrast Inversion in Noncontact Atomic Force Microscopy Imaging of Adsorbates. Phys. Rev. B 2008, 77, 195410. 